\title{
Functional Nanomaterials
}

\section{Habib M. Pathan, ${ }^{1}$ Rajaram S. Mane, ${ }^{2}$ Yahya A. Ismail, ${ }^{3}$ Angaiah Subramania, ${ }^{4}$ Wegdan Ramadan, ${ }^{5}$ and Sharad D. Bhagat $^{6}$}

\author{
${ }^{1}$ Advanced Physics Laboratory, Department of Physics, University of Pune, Pune 411007, India \\ ${ }^{2}$ Center for Nanomaterials and Energy Devices, School of Physical Sciences, Swami Ramanand Teerth Marathwada University, \\ Nanded 431606, India \\ ${ }^{3}$ Department of Biological Sciences and Chemistry, University of Nizwa, Nizwa 616, Oman \\ ${ }^{4}$ Centre for Nanoscience and Technology, Pondicherry University, Puducherry 605014, India \\ ${ }^{5}$ Department of Physics, Alexandria University, Alexandria, Egypt \\ ${ }^{6}$ Department of Chemistry and Biochemistry, Florida State University, Tallahassee, FL 32306, USA
}

Correspondence should be addressed to Habib M. Pathan, pathan@physics.unipune.ac.in

Received 6 May 2012; Accepted 6 May 2012

Copyright (C) 2012 Habib M. Pathan et al. This is an open access article distributed under the Creative Commons Attribution License, which permits unrestricted use, distribution, and reproduction in any medium, provided the original work is properly cited.

The impetus of scientific community around the world lately has been on nanoscience and technology as the researchers have envisioned great many applications for these materials earning them rightly the name "functional nanomaterials." This domain of materials is special since they display extraordinary transitions in their properties in their size regime as compared to their bulk counterparts. The electrical, electronic, optical, thermal, mechanical, or magnetic properties of these classes of materials have opened great avenues for many important applications and redefined and improved the efficiencies of the devices generated out of them.

The novel era of functional nanomaterials has goaded change in the face as well as phase of all the materials and facilitated design of materials customized to the needs of the mankind by manipulating matter at the nanoscopic level.

The world is and will keep on witnessing the demand for new nanomaterials for multifarious high-tech and industrial applications. This special issue is aimed at bringing out an overview of the latest advances in the field of nanomaterials with special emphasis on synthesis and applications highlighting greater strides in the field. Nanoscience and technology being an interdisciplinary field encompasses a wide range of subjects like physics, chemistry, biology, biotechnology, engineering, mathematics, and so forth. In view of this the present issue broadly covers carbonbased, nanomaterials, their synthesis and applications, wide bandgap semiconductors, and their applications as nanomaterials in sensing, photocatalysis and photovoltaics.

The present issue covers syntheses of titania reported by Cloutier and coworkers and Uekawa et al. of which the former deals with facile preparation of porous titania nanostructures and their role in the enhanced power conversion in heterojunction solar cells. The latter presents a large surface area $\mathrm{TiO}_{2}$ nanoparticles' synthesis through hydrolysis. Another wide bandgap semiconductor, the richest family of nanostructures among all materials, the much talked about $\mathrm{ZnO}$, is also accommodated. Tseng et al. employed various polyol solvents to synthesize zinc oxide polycrystalline nanostructures in the form of fibers (1D), rhombic flakes (2D), and spheres (3D). Thus formed $\mathrm{ZnO}$ structures were applied in the degradation of methylene blue to compare their photocatalytic efficiency.

Keeping in view the promising applications of carbon nanotubes (CNTs), two papers are included on CNTs. Zhao and $\mathrm{Li}$ reported an improved layer-by-layer vacuum filtration method for the fabrication of single-walled carbon nanotube (SWCNT) films aiming at a series of SWCNT films with controllable thickness and density. The electrical transport properties of the multi-layered SWCNT films have been investigated. Mazov et al. present the simple procedure of the vapor-phase bromination of multiwall carbon nanotubes (MWNTs) at moderate temperatures. MWNTs with average 
diameter $9 \pm 3 \mathrm{~nm}$ were treated with bromine vapours to produce Br-functionalized products.

Habib M. Pathan

Rajaram S. Mane

Yahya A. Ismail

Angaiah Subramania

Wegdan Ramadan

Sharad D. Bhagat 

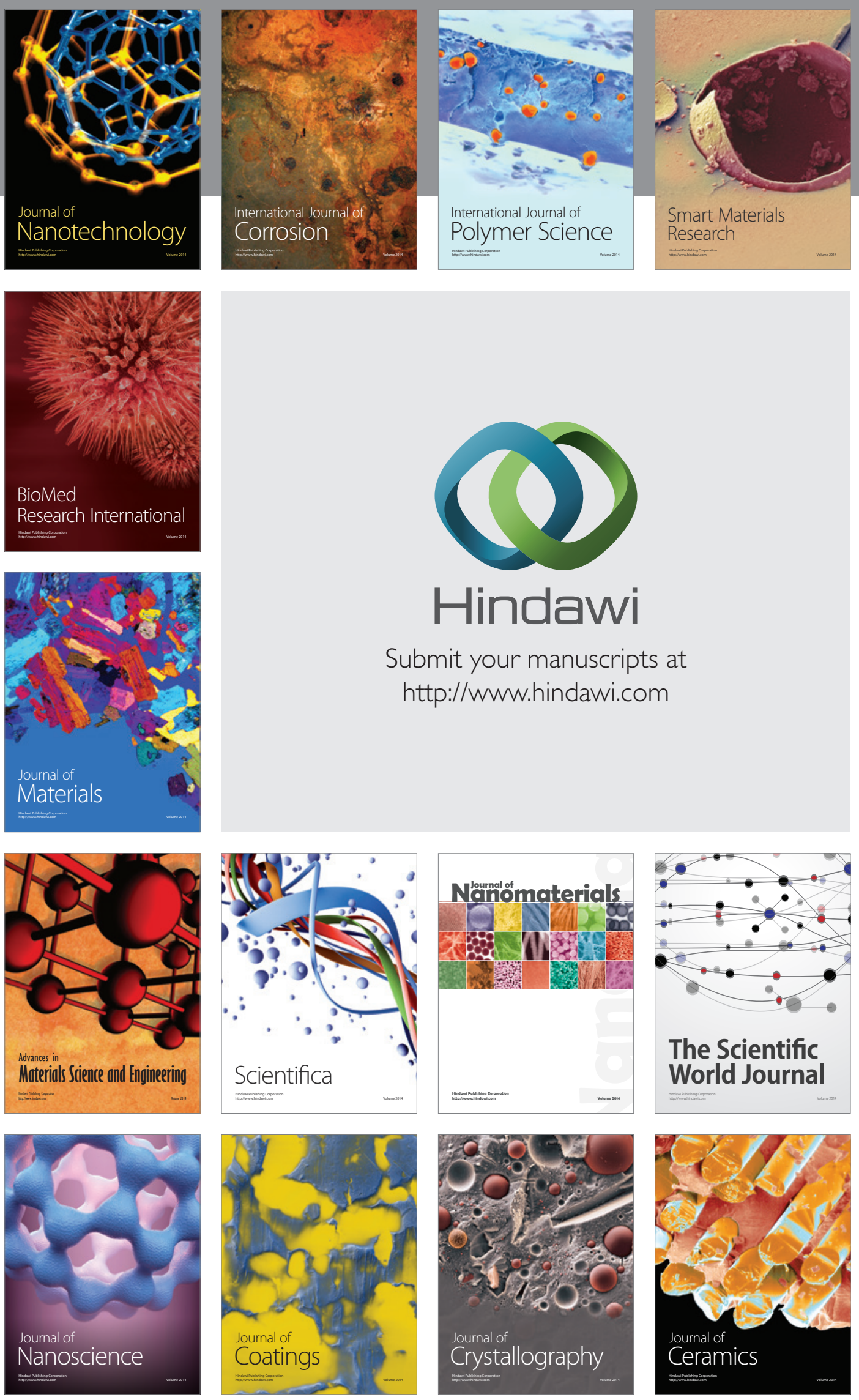

The Scientific World Journal

Submit your manuscripts at

http://www.hindawi.com

\section{World Journal}

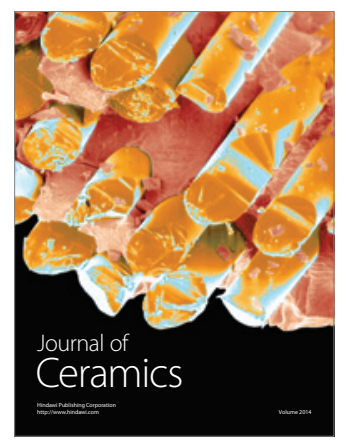

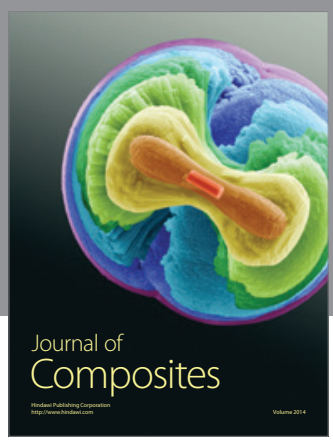
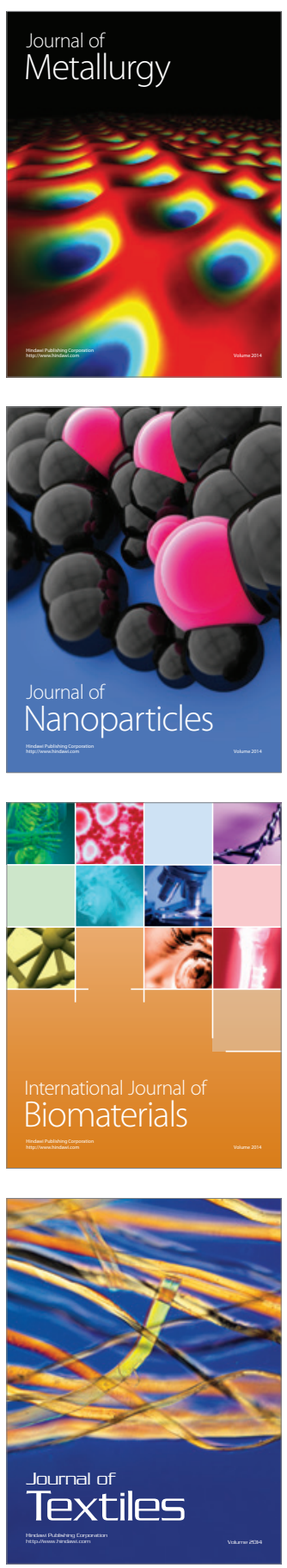